\title{
弦向掠叶片对动叶可调轴流风机性能影响模拟
}

\author{
李春㬢 范福伟 刘宏凯 叶学民 \\ (华北电力大学电站设备状态监测与控制教育部重点试验室 保定 071003)
}

\begin{abstract}
摘要: 叶片弦向弯掠技术是提升轴流风机气动性能的有效手段。针对带后置导叶的 OB-84 型单级动叶可调轴流风机, 利用 Ansys 数值软件, 对比叶片掠设计前后风机的性能曲线和内流特征, 开展静力结构特性分析并进行了噪声预估, 探究了前掠 角度在不同动叶安装角下的影响。结果表明: 设计安装角下, 前掠叶片能有效提高叶轮做功能力和导叶扩压能力, 减少泄漏 损失, 提升风机整体性能, 在小流量侧和大流量侧效果更明显; 设计流量下前掠 $8.3^{\circ}$ 性能最优, 全压和效率分别提升 $2.1 \%$ 和 $1.68 \%$, 但气动噪声有所增大; 叶片掠向改型后虽变形增大, 但仍满足材料强度要求; 在变安装角下, 前掠叶片风机性能均 有所降低。设计安装角下所得最佳前掠角度适用于长期带基本负荷的轴流风机。
\end{abstract}

关键词: 动叶可调轴流风机; 弦向掠叶片; 气动性能; 静力结构分析; 噪声预估

中图分类号: TM621

\section{Simulation on Aerodynamic Performance of a Variable-pitch Axial Flow Fan with Chordwise Swept Blades}

\author{
LI Chunxi FAN Fuwei LIU Hongkai YE Xuemin \\ (Key Lab of Condition Monitoring and Control for Power Plant Equipment of Ministry of Education, \\ North China Electric Power University, Baoding 071003)
}

\begin{abstract}
The chordwise swept blades is an effective way to improve the aerodynamic performance of large axial fans. For a single-stage variable-pitch axial fan of type OB-84 with rear guide vanes, the aerodynamic performance and internal dynamics of the fan before and after blade sweeping are simulated with Ansys software, the static structure features are investigated, and the noise is predicted, as well as the influence of the sweep angle at different blade angles is examined. Simulated results show that under the design blade angle, the forward-swept blade can effectively raise the working ability of impeller and pressure recovery ability of guide vanes, reduce the leakage loss, and then the fan performance is improved, especially in the small and large flow rate. The forward-swept angle of $8.3^{\circ}$ has the best performance at the design point and the total pressure and efficiency are increased by $2.1 \%$ and $1.68 \%$, but the noise is increased. After the blades swept, the aggravated deformation still meets the requirements of material strength. The performance of the forward swept blade fan is reduced under variable blade angle conditions, so the best forward-swept angle obtained at the design blade angle is suitable for fans operating at rated loads.
\end{abstract}

Key words: variable-pitch axial fan; chord swept blades; aerodynamic performance; static structural analysis; noise prediction

\section{0 前言}

动叶可调轴流风机具有整体性能高、流量大和 易调节等优点被电站广泛应用, 但其耗电量占厂电 量约 30\%, 且气动噪声较大, 因此开展此类风机的 气动和声学性能研究对于响应国家节能减排战略、 降低环境噪声具有重要的现实意义。

轴流风机性能受流道内多种流动因素影响, 包

* 河北省自然科学基金(E2016502098)和中央高校基本科研业务费专项基 金(13MS98)资助项目。20181014 收到初稿, 20190325 收到修改稿
括通道内涡流、叶尖泄漏流、叶片边界层和壁面边 界层, 减少与这些流动因素相关的损失, 对性能的 改善至关重要。叶片弦向弯掠技术是实现性能改善 的重要径，并在轴流风机和压气机方面取得了积极 进展。在轴流风机方面, MOHAMMED 等 ${ }^{[1]}$ 和 WADIA 等 ${ }^{[2]}$ 基于试验方法研究了小弦向掠角叶片 和原叶片轴流风机的性能, 指出弦向前掠叶片可有 效提高风机的全压、效率，尤其是低于设计流量下。 金永平 ${ }^{[3]}$ 采用回归拟合方法研究弯掠叶片对矿用对 旋式轴流通风机的影响, 得出第一、二级最佳前掠 角分别为 $9.4^{\circ}$ 和 $7.84^{\circ}$, 风机效率提高约为 $1.64 \%$ 。 
PATEL 等 $^{[4]}$ 对某一低速轴流风机采用非自由浴设 计, 得到前掠 $10^{\circ}$ 叶片, 并局部扩展尖端轮廓, 表 明前掠改型后, 风机失速裕度和总压升系数提高 $1.95 \%$ 和 $3.6 \%$, 效率和全压提升方面略有改善, 在 高流量系数下, 所有转子的效率相差不大。IIIKAN 和 $A Y D E R^{[5]}$ 研究了 $30^{\circ}$ 和 $45^{\circ}$ 前掠和后掠叶片对低 压轴流风机性能的影响, 指出前掠和后掠叶片未能 有效提升风机性能, 但在设计流量左侧的失速点附 近, 前掠叶片的总压升系数提升约 $6 \%$, 后掠叶片 则显著下降 $18 \%$, 效率变化与全压类似, 且掠对叶 尖涡流有一定影响, 可作为控制二次流损失的工具。 MASI 等 ${ }^{[6]}$ 对一低轮毂比轴流风机采取叶片前掠 $45^{\circ}$ 和后掠 $45^{\circ}$ 改型设计, 指出前掠设计在整个运行范 围内全压较原风机均有提升, 提升量从 $1 \% \sim 4 \%$, 效率则变化不大; 设计流量下节流时的失速裕度增 加 $10 \%$ 以上。GIESECKE 等 ${ }^{[7]}$ 针对机车冷却系统用 轴流式风机, 基于自由浴设计准则, 对转子和定子 均采用前掠设计, 使效率提升至 $82 \%$, 喘振裕度提 高 $22 \%$ ，转子效率提高 $15 \%$, 噪声降低 $5.1 \mathrm{~dB}$ 。

压气机方面, CUI 等 ${ }^{[8]}$ 指出前掠和后掠均有利 于叶片流动特性的改善, 而掠叶片也会影响流体的 入流条件、三维冲击结构和内部载荷分布, 前掠引 起的流场中新的径向平衡和低能流体在边界层中的 输运均有利于减小转子区的激波和阻塞, 同时, 叶 尖区前缘附近的叶片负荷减小, 前掠转子中的泄漏 流强度受到抑制，其失速裕度增长超过 $10 \%$ 。 BENINI 等 ${ }^{[9]}$ 针对某跨声速轴向低压气机设计了 26 种前掠和后掠转子方案, 表明后掠转子的压气机特 性优于前掠转子, 该发现与传统的掠叶片研究成果 相反, 但掠叶片可使得气流与叶片更容易分离, 减 少冲击损失和改善空气动力性能。昌皓等 ${ }^{[10]}$ 选择 NASA Rotor 37 作为基准模型, 设计前后掠角分别 为 $10^{\circ}$ 和 $20^{\circ}$ 叶片, 模拟表明, 相较于原叶片, 前掠 导致入口气流攻角减小, 叶片负荷减小, 后掠叶片 反之, 且在小流量侧影响显著, 但前掠会加剧进口 的周向脉动。宋彦萍等 ${ }^{[11]}$ 利用数值和试验方法研究 了前掠对压气机端壁流动的影响, 指出叶片前掠改 变了三维压力场, 降低了端壁损失与通道涡的强度 和尺度, 加强了对端区低能流体的抽吸作用, 但增 加了叶片中部损失。

上述研究表明, 采用弦向弯掠叶片可有效提高 性能, 且对于低压低速风机和压气机的研究中, 普 遍认为前掠较后掠设计效果突出, 但对气动噪声研 究较少。而对于大型动叶可调轴流风机, 其结构有 诸多不同，如不仅多采用高效的大尺寸三维扭叶片， 且该类型风机转速高、调节范围广, 并采用后置导
叶稳定流场以进一步提高效率，但目前针对动叶可 调轴流风机实施叶片弦向掠改型的研究甚少。为此, 本文以 OB-84 型单级动叶可调轴流风机为对象, 对 动叶片实施一定范围角度弦向掠改型, 基于数值计 算模拟叶片改型后的轴流风机性能和噪声, 开展静 力结构特性分析, 并对比不同动叶安装角下的直叶 片和掠叶片风机的性能曲线, 为大型动叶可调轴流 风机应用叶片弯掠技术提供理论依据。

\section{1 数值计算方法}

\section{1 计算模型}

研究对象为 OB-84 型带后置导叶单级动叶可调 轴流风机。模型如图 1 所示, 设计参数见表 1 。

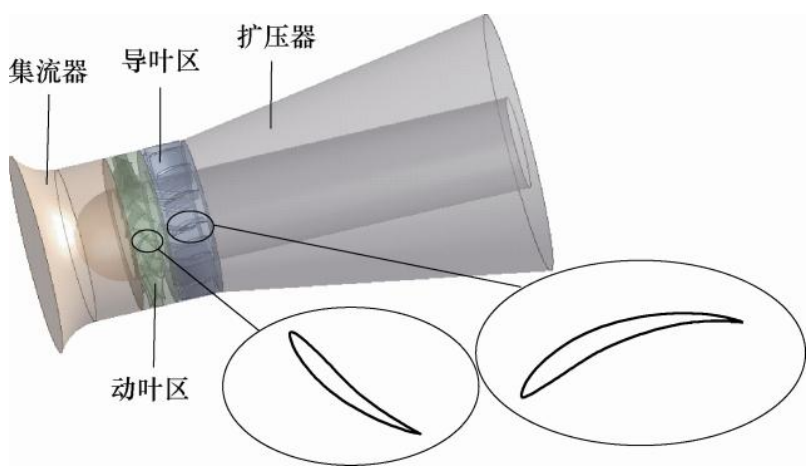

图 1 OB-84 型单级动叶可调轴流风机模型

表 1 风机设计参数

\begin{tabular}{lc}
\hline \multicolumn{1}{c}{ 设计参数 } & 数值 \\
\hline 动叶片数目 & 14 \\
导叶片数目 & 15 \\
叶轮直径 $/ \mathrm{mm}$ & 1500 \\
轮教比 & 0.6 \\
叶顶间隙 $/ \mathrm{mm}$ & 4.5 \\
额定转速 $/(\mathrm{r} / \mathrm{min})$ & 1200 \\
体积流量 $($ 设计工况 $) /\left(\mathrm{m}^{3} / \mathrm{s}\right)$ & 37.14 \\
全压 $($ 设计工况 $) P_{\mathrm{t}} / \mathrm{Pa}$ & 2348 \\
动叶安装角 $\beta /\left({ }^{\circ}\right)$ & 32 \\
\hline
\end{tabular}

采用 Gambit 软件建立模型, 原叶片为常规径向 叶片，改型后为弦向掠叶片，如图 2 所示。鉴于大 角度弯掠叶片加工不便 ${ }^{[12]}$, 且前掠叶片比后掠叶片 的风机整体性能更佳 ${ }^{[1-3,5-6,8]}$, 为此下文采用前掠设 计，定义掠角为 $\delta$, 采用 $\delta=2.0 、 4.0 、 6.0 、 8.3 、 10.0^{\circ}$ 开展分析, 所选角度在文献[3-4]范围附近并进行适 当加大, $\delta=0$ 为原叶片。前掠叶片设计时保持 $\delta=0$ 叶片三维尺寸不变, 通过引入弦向掠角度, 改变叶 片重心积迭线而得到。原叶片和前掠叶片重心积迭 线的形状与掠角如图 3 所示，其中重心积迭线采用 根部直线段+顶部圆弧段, 分界点 $D$ 位于 0.4 相对叶 
高处; 轮㺉轴心和积迭线末端连线 $O S$ 与原叶片积 迭线 $R H$ 间的夹角 $\delta$ 为掠角, 弦向前掠规定为叶片 截面中弧线与重心积迭线交点处切线指向叶片前缘 方向, 掠角为正值。
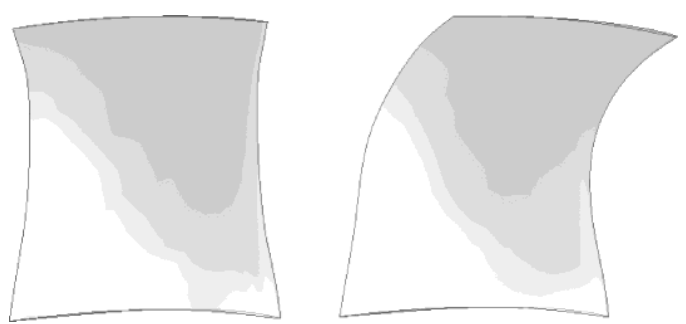

图 2 原叶片与前掠叶片

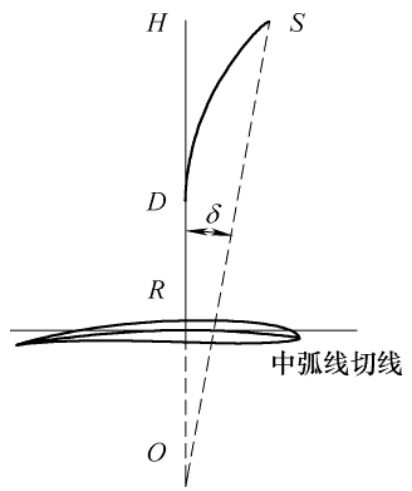

图 3 重心积迭线与前掠角度示意图

考虑到三维扭叶片和叶顶附近流动的复杂性, 叶顶截面划分三角形网格, 吸/压力面划分四方网 格, 壁区设置标准壁面函数, 并利用尺寸函数进行 加密, 划分结构化/非结构化混合网格; 其余区域的 网格划分参照动叶区网格划分, 亦采用结构化/非结 构化混合网格。为验证计算精度的网格无关性, 采 取网格数为 382 万、 426 万、 465 万和 533 万并进行 模拟对比, 如表 2 所示, 考虑计算精度和计算时长, 最终选取整机网格数为 465 万。

表 2 网格无关性验证

\begin{tabular}{cccc}
\hline 网格数/万 & 全压 $P_{\mathrm{t}} / \mathrm{Pa}$ & 效率 $\eta(\%)$ & 计算时长 $/ \mathrm{h}$ \\
\hline 382 & 2316.43 & 81.052 & 9.8 \\
426 & 2325.95 & 81.237 & 10.2 \\
465 & 2333.55 & 81.506 & 10.6 \\
533 & 2335.72 & 81.523 & 11.5 \\
\hline
\end{tabular}

\section{2 计算方法及边界条件}

采用 Fluent 对原风机和掠叶片风机进行数值模 拟, 流场设置如下。

(1) 控制方程: 采用三维定常雷诺时均方程和 连续方程, 方程中各项均采用二阶迎风格式进行 离散。

（2）湍流模型：选用能更好地模拟叶顶间隙内 的复杂流动和有效解决旋转运动的 Realizable $k-\varepsilon$
模型 ${ }^{[13]}$, 基于 SIMPLE 算法实现速度与压力的耦合。

(3) 区域划分：模拟流场包含整机的四个区域, 定义动叶区为旋转区, 给定转速和旋转方向, 其余 区域为静止区。

(4) 面域定义: 集流器进口截面和扩压器出口 截面为整个流场的进、出口, 分别采用 velocity 入 口和 outflow 出口条件; 进口湍动能和湍动能耗散 率由流体平均流速和截面特征长度代入经验公式计 算得到 ${ }^{[14]}$; 动叶片和动叶轮勃表面为旋转面, 其余 为静止面; 各区域交界面定义 Interface 并进行耦合 用于数据传递和交换; 所有壁面采用无滑移边界条 件, 近壁区条件给定标准壁面函数。

模拟中忽略重力和壁面粗粘度的影响。当进出 口各方向速度、 $k$ 和 $\varepsilon$ 等参数的残差小于 $10^{-4}$ 时, 视为模拟计算达到稳态收敛。

\section{3 静力结构分析}

在 ANSYS 有限元分析软件中, 将模拟数据导 入静力分析模块, 使流场计算出的压力结果与结构 场计算过程进行数据交换和耦合 ${ }^{[15]}$, 由此分析不同 掠角度下叶片的等效应力和变形。计算中，采用非 结构化混合网格方式划分叶轮实体网格; 轮毂及叶 片材料选用铝合金; 叶轮施加旋转产生的离心力载 荷、空气对叶片和轮鈠表面的压力载荷及自身所受 地球重力加速度载荷, 其中压力载荷垂直于叶片表 面, 对叶片的作用主要沿轴向, 产生弯曲变形, 离 心力载荷沿叶片径向分布, 产生拉伸变形 ${ }^{[16]}$ 。

\section{4 模拟结果验证}

图 4 对比了风机模拟值与样本值。图中量纲一 流量系数定义为: $Q=Q_{\mathrm{v}} /(A u)$, 其中, $Q_{\mathrm{v}}$ 为体积流 量, $A$ 为截面面积, $u$ 为叶顶圆周速度。定义全压 $P_{\mathrm{t}}=P_{\text {out }}-P_{\text {in }}$, 效率 $\eta=P_{\mathrm{e}} / P_{\mathrm{sh}}, P_{\text {in }}$ 和 $P_{\text {out }}$ 为风机进出口 截面的总压, $P_{\mathrm{e}}$ 为风机轴功率, $P_{\mathrm{sh}}$ 为有效功率。由 图 4 可知, 掠叶片风机较原风机的全压和效率平均 偏差为 $2.69 \%$ 和 $1.04 \%$, 设计工况下的偏差为 $1.88 \%$ 和 $1.80 \%$, 均在允许误差范围内, 说明模拟结果

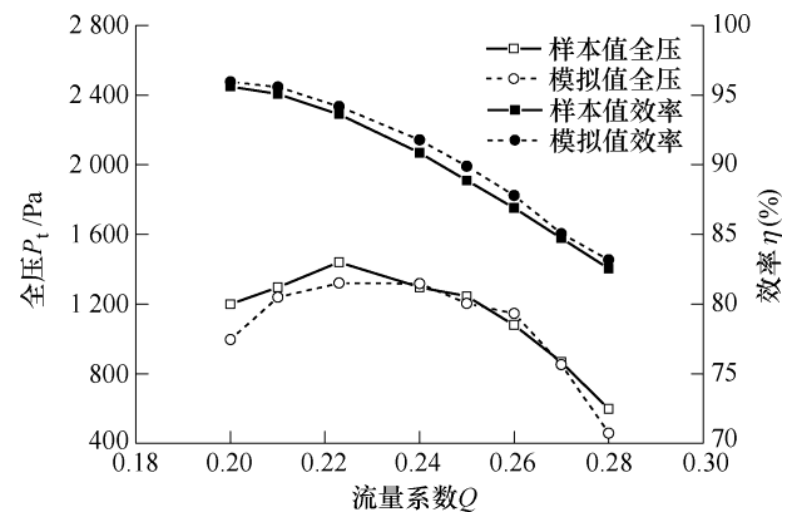

图 4 模拟结果与样本值对比 
可信度较高, 所建模型可反映该风机的实际运行 状况。

\section{2 模拟结果及分析}

\section{1 气动性能}

图 5 为前掠角度对风机全压和效率的影响。图 $5 \mathrm{a}$ 表明, 设计工况下, 随前掠角度 $\delta$ 增大, 全压和 效率变化趋势一致, 呈的变化, 在 $\delta=6.0^{\circ} \sim 8.3^{\circ}$ 时全压有明显提高, 且均高于 $\delta=0$ 情形; 在 $\delta=4.0^{\circ} \sim$ $6.0^{\circ}$ 时效率较原模型效率偏差很小, 平均偏差仅为

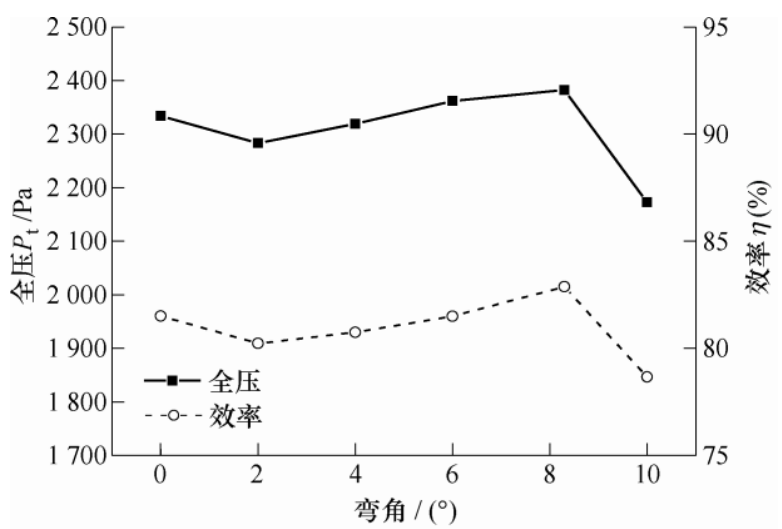

(a) 设计工况下

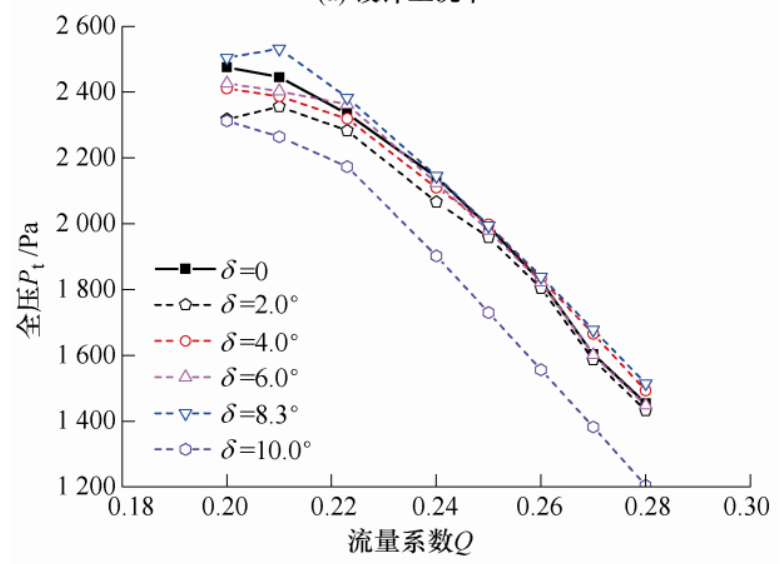

(b) 不同流量下的风机全压变化

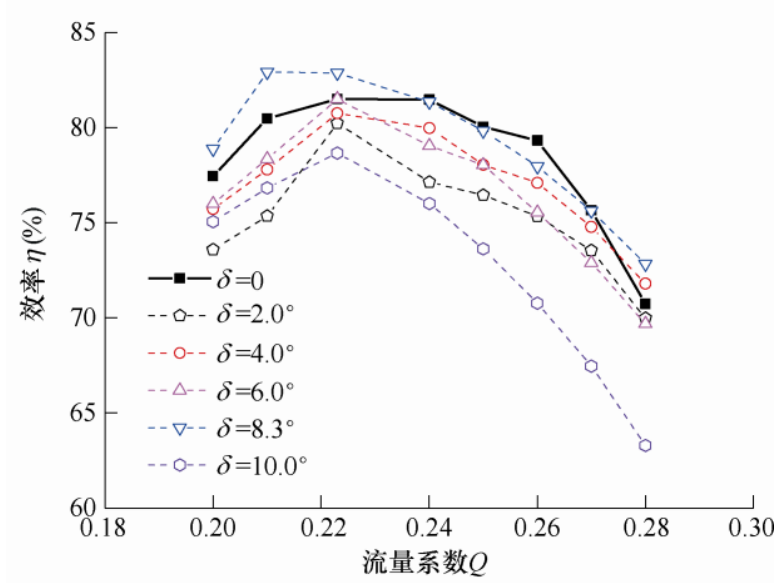

(c) 不同流量下的风机效率变化

图 5 前掠叶片对风机性能曲线的影响
$0.475 \%$; 在 $\delta=8.3^{\circ}$ 时全压和效率达到最大, 与原叶 片相比, 分别提高约 $2.1 \%$ 和 $1.68 \%$; 当 $\delta>10.0^{\circ}$ 后 全压骤降。综合考虑前掠角度对全压和效率的影响, 设计工况下的最佳前掠角度范围为 $6.0^{\circ} \sim 8.3^{\circ}$, 与文 献[3-4]结论接近。

图 $5 \mathrm{~b}$ 和 $5 \mathrm{c}$ 为不同流量下前掠角度对风机性能 的影响。其影响在不同范围内呈现不同特征，这表 现在: $\delta=8.3^{\circ}$ 时, $Q=0.2 \sim 0.24$ 时(含设计工况点)全 压和效率较原风机显著提高; $Q=0.24 \sim 0.26$ 时全压 提升不明显, $Q>0.26$ 时全压提升明显; $Q=0.24 \sim 0.27$ 效率较原风机降低, $Q>0.27$ 时效率提升明显; 三 种情形下全压平均提高 $2.26 \% 、 0.24 \% 、 4.46 \%$ ， 效率变化分别为 $2.19 \% 、-0.75 \% 、 2.98 \% 。 \delta=4.0^{\circ}$ 和 $6.0^{\circ}$ 时, 小流量范围全压较原风机有所降低, 随 流量提高, 全压逐渐高于原风机情形, 但全流量 范围内效率均有所下降，全压和效率平均变化幅 度分别为 $0.27 \% 、 0.78 \%$ 和 $-1.85 \% 、-2.69 \%$; 其余 前掠叶片下的风机全压效率在全流量范围较原风 机下降明显。由此可见, 在小流量侧和大流量侧, 前掠叶片对全压和效率提升较大, 性能提升效果 更明显。

为揭示上述气动性能的内在变化, 下文对内流 特征开展分析。由图 $5 \mathrm{a}$ 可知, $\delta=6.0^{\circ} \sim 8.3^{\circ}$ 时全压 和效率均处于增大趋势且在 $\delta=8.3^{\circ}$ 处达到峰值; 另 外, 风机选型时通常选择较大的参数裕量 ${ }^{[17]}$, 致使 风机实际运行时流量多处于设计点左侧, 且 $\delta=6.0^{\circ}$ 和 $8.3^{\circ}$ 较其他前掠角度在设计点提升显著, 因此下 文分析原风机和 $\delta=6.0^{\circ}$ 及 $8.3^{\circ}$ 时风机在设计工况下 的总压升系数、扩压系数沿叶高变化和叶顶截面熵 产率分布特征。

\section{2 总压升系数和扩压系数}

动叶区总压升系数和导叶区扩压系数可表征动 叶轮做功能力和导叶扩压能力的强弱。图 6 为原风 机、 $\delta=6.0^{\circ}$ 和 $8.3^{\circ}$ 叶片风机的动叶区总压升系数和导 叶区扩压系数沿叶高分布。定义相对叶高 $R=\left(r-r_{\mathrm{h}}\right) / r_{t}$,

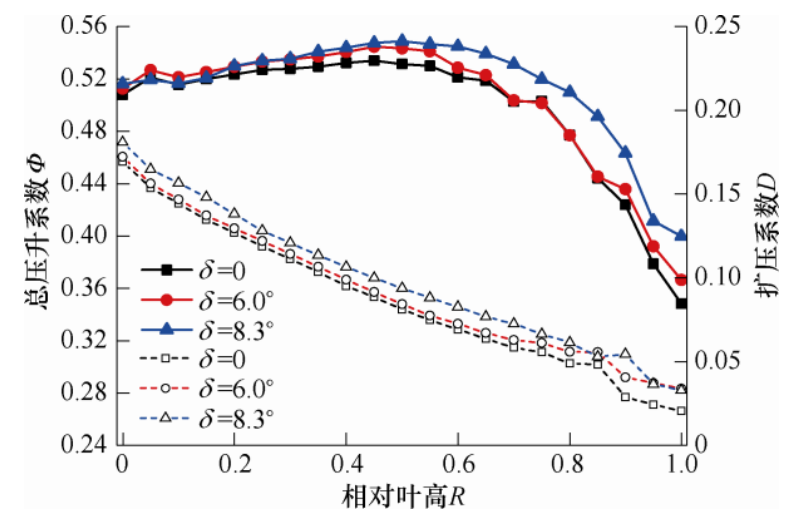

图 6 总压升系数和扩压系数沿叶高变化 
总压升系数 $\Phi=2\left(P_{2 \mathrm{t}}-P_{1 \mathrm{t}}\right) / \rho u^{2}$, 扩压系数 $D=2\left(P_{2 \mathrm{~s}}-\right.$ $\left.P_{1 \mathrm{~s}}\right) / \rho u^{2}$, 其中, $r$ 为径向叶高, $r_{\mathrm{h}}$ 和 $r_{\mathrm{t}}$ 为轮勃和叶 轮半径, $P_{1 \mathrm{t}}$ 和 $P_{2 \mathrm{t}}$ 为动叶进出口总压, $P_{1 \mathrm{~s}}$ 和 $P_{2 \mathrm{~s}}$ 为 导叶进出口静压, $\rho$ 为气体密度。

由图 6 可知, $R=0 \sim 0.8$ 范围内总压升系数相对 较高, 说明此叶高范围为叶片主要做功区, $R=0.8 \sim$ 1 为叶顶附近, 由于气流叶顶泄漏导致做功能力下 降, 总压升系数迅速减小; $\delta=8.3^{\circ}$ 叶片风机在全叶 高范围总压升系数较原风机提高, 尤其在叶片上半 部及顶端, 表明 $\delta=8.3^{\circ}$ 能有效抑制叶顶间隙泄漏流 的产生; $\delta=6.0^{\circ}$ 叶片风机在 $R=0 \sim 0.65$ 和 $R=0.85 \sim 1$ 范围内总压升系数有所提高, 而在 $R=0.65 \sim 0.85$ 内 变化不明显, 说明 $\delta=6.0^{\circ}$ 时加强了叶片中下部做功 能力, 抑制叶顶泄漏流效果不明显。导叶区扩压系 数总体沿叶高呈单调递减趋势, 在 $R=0 \sim 0.8$ 范围 内, $\delta=8.3^{\circ}$ 叶片风机导叶扩压能力最强, $\delta=6.0^{\circ}$ 次之; $R=0.8 \sim 1$ 范围内, 两种掠角风机导叶扩压系数相 差不大, 但全叶高范围均优于原风机。综上所述, $\delta=6.0^{\circ}$ 和 $8.3^{\circ}$ 叶片均能有效提高叶轮做功能力和导 叶扩压能力, 降低叶顶泄漏损失, 但后者在总体上 优于前者。

\section{3 叶顶区熵产率分布}

熵产率可反映能量耗损的程度, 即熵产率增加, 流动损失加剧 ${ }^{[18]}$ 。风机内部流场复杂, 尤其动叶区 流道内气流速度差较大, 而温度基本不变, 因此以 速度耗散所产生的熵产为主。该熵产主要包括粘性 耗散和湍流耗散, 且后者引起的熵产较前者要高两 个数量级 ${ }^{[14]}$ 。

图 7 为动叶区叶顶截面处的熵产率分布。叶片 附近熵产率远大于流道内熵产率; 叶顶前端商产率 较小, 但叶片前掠设计后, 熵产率由 $200 \mathrm{~W} \cdot \mathrm{m}^{-3} \cdot \mathrm{K}^{-1}$ 增至 $400 \mathrm{~W} \cdot \mathrm{m}^{-3} \cdot \mathrm{K}^{-1}$, 这是因叶片前掠后叶顶前 缘与气流冲击作用加剧, 冲击损失增加; 叶顶泄漏 的存在使得叶顶吸/压力面侧附近流场熵产率远大 于动叶区其他区域且梯度分布密度较大, 并在泄漏 涡作用下压力面摘产率小于吸力面, 吸力面侧梯度 分布稀疏, 逐渐向叶顶后部流域直至动叶出口和相 邻叶片压力面延伸。 $\delta=6.0^{\circ}$ 和 $8.3^{\circ}$ 较原风机叶顶吸/ 压力面侧熵产率明显降低, 但 $\delta=6.0^{\circ}$ 时高熵产率区 域分布与原叶片相似, 集中在叶顶中后部, 而 $\delta=8.3^{\circ}$ 时高熵产率区域则集中在叶顶靠近尾缘处, 说明 $\delta=8.3^{\circ}$ 时较其余两种方案延迟了叶顶泄漏现象的发 生, 从而减少泄漏损失, 与图 6 结论相对应, 证实 了叶片前掠设计对叶顶泄漏的影响。
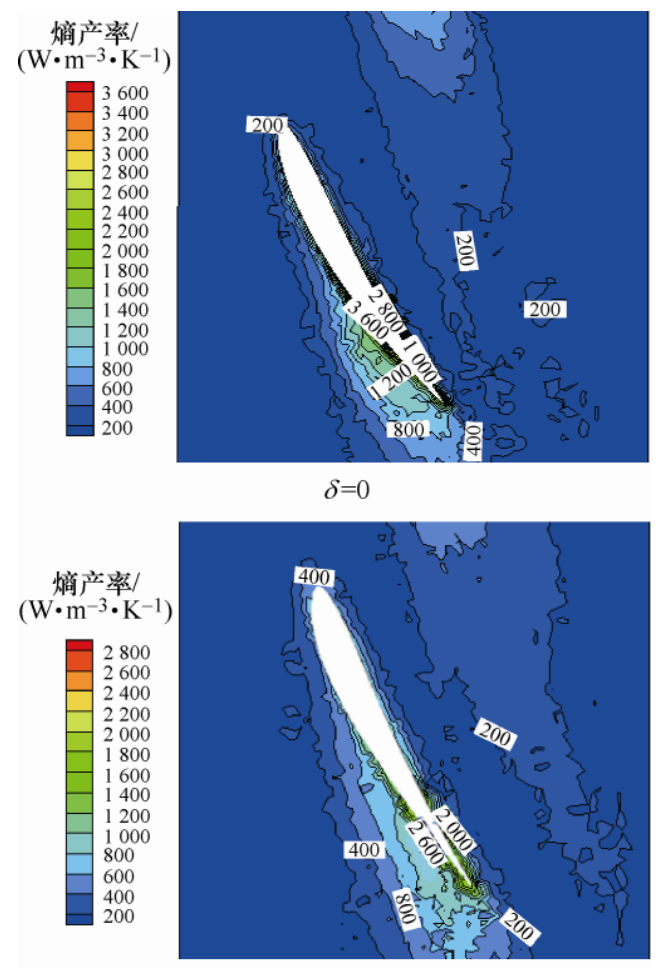

$\delta=6.0^{\circ}$

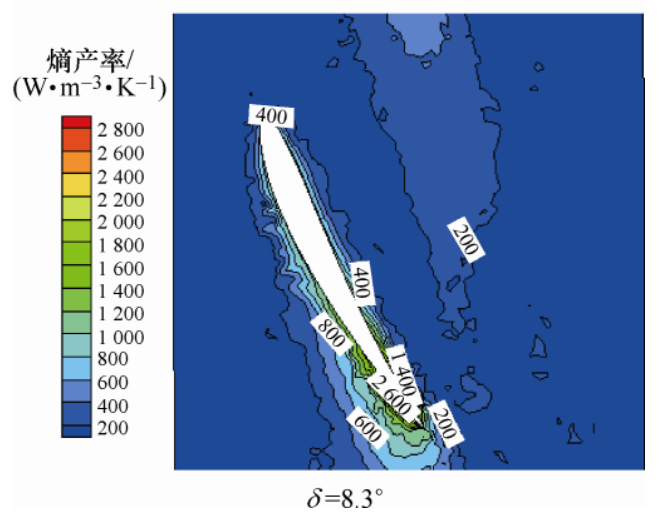

图 7 动叶区叶顶截面熵产率分布

\section{4 静力结构特性}

为分析前掠角度对叶片表面载荷分布的影响, 图 8 分别给出了叶片在不同前掠角度下的吸/压力面 等效应力分布, 由 Drucker-Prager 屈服准则定义等 效应力为 $\sigma_{\mathrm{e}}=a+b \cdot \sigma_{\mathrm{m}}$, 其中 $\sigma_{\mathrm{m}}$ 为流体静应力, $a$ 和 $b$ 常数。 $\delta=0$ 时压力面中部的等效应力最大, 从中部 向四周逐渐减小, 吸力面上的等效应力由叶根中后 部至叶顶区呈下降趋势, 最小等效应力主要分布在 叶顶区。前掠叶片等效应力分布较原叶片有明显不 同, 压力面上等效应力由叶片前缘中下部至叶顶和 尾缘逐渐减小, 吸力面上由叶片前缘中下部和叶根 部至叶顶区逐渐减小; 且由表 3 可知, 前掠叶片最 大等效应力和最小等效应力较原叶片提高, 这是因 叶片的前掠设计使顶部前缘与气流冲击剧烈, 前缘 气流沿叶片表面向中下部集中, 导致压力面前缘中 下部流量增加, 负载加大, 因气流对叶片前缘上半 
部的冲击作用而产生的径向分力与叶片所受离心力 和自身重力的共同作用, 使叶片有被沿径向拉伸变

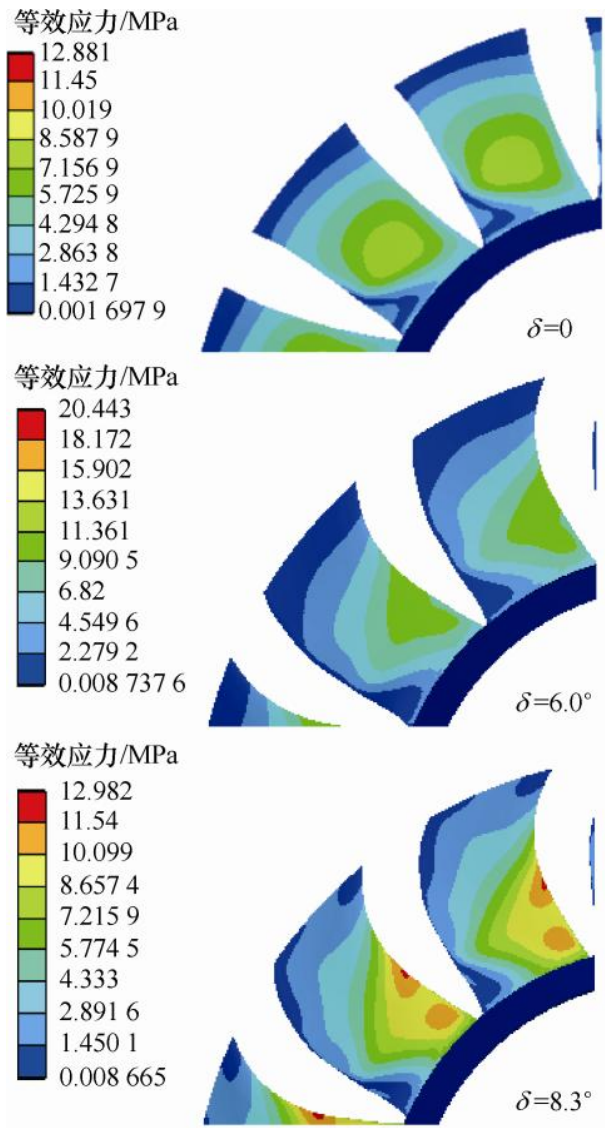

(a) 压力面等效应力
形的姿态 ${ }^{[16]}$, 加大吸/压力面前缘中下部和根部载 荷，等效应力变大。

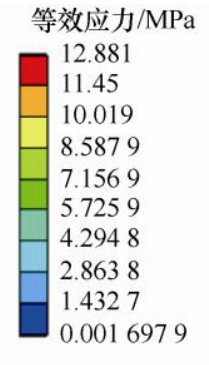

等效应力 $/ \mathrm{MPa}$

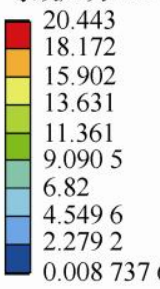

等效应力 $/ \mathrm{MPa}$

$\square=12.982$

11.54
10.099

8.6574

7.2159

$-5.7745$

4.333

2.8916

1.4501
0.008665
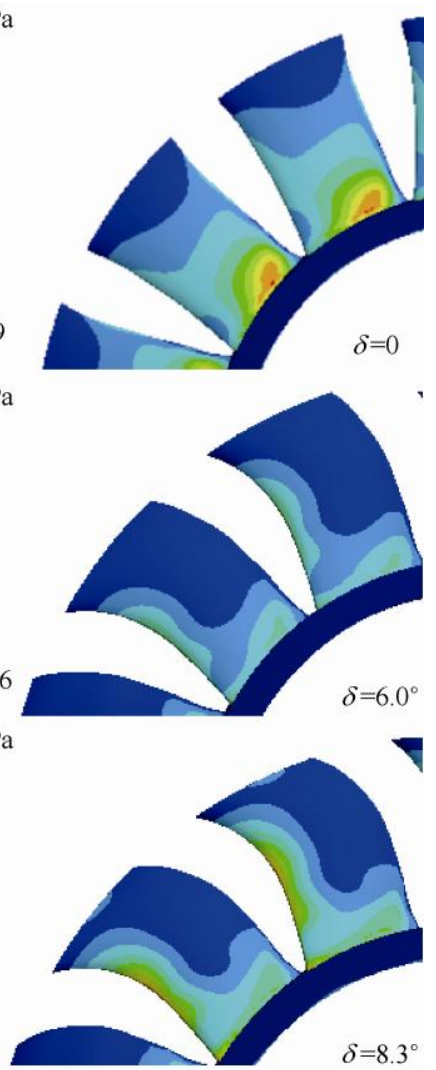

(b) 吸力面等效应力

图 8 前掠角度对等效应力分布的影响

表 3 叶片等效应力

\begin{tabular}{ccc}
\hline 掠角度 $/\left(^{\circ}\right)$ & 最小等效应力 $/ \mathrm{MPa}$ & 最大等效应力 $/ \mathrm{MPa}$ \\
\hline$\delta=0$ & 0.0016979 & 12.881 \\
$\delta=6.0$ & 0.0087376 & 20.443 \\
$\delta=8.3$ & 0.008665 & 12.982 \\
\hline
\end{tabular}

由图 9 叶片变形和表 4 叶片最大变形量可知, 原叶片叶片尾缘中上部和前缘顶部变形量较大, 至 叶根部分最小为零, 最大变形量为 $0.076 \mathrm{~mm}$; 而前 掠叶片最大变形发生在叶顶前缘且最大变形量较原 叶片加大, 这是因叶片前掠改型后, 叶片顶端前缘 与气流的冲击作用加剧; $\delta=6.0^{\circ}$ 时最大变形量是三 种方案中最大, $\delta=8.3^{\circ}$ 时有所减小, 变形相对减弱, 变形量由叶顶前缘沿叶片对角线至叶根部分逐渐减 小为零。上述结果表明, 叶片掠改型后变形较原叶 片加剧, 需对其进行静强度校核。

表 4 叶片最大变形量

\begin{tabular}{cc}
\hline 掠角度 $/\left(^{\circ}\right)$ & 最大变形量 $/ \mathrm{mm}$ \\
\hline$\delta=0$ & 0.076 \\
$\delta=6.0$ & 0.14 \\
$\delta=8.3$ & 0.13 \\
\hline
\end{tabular}

在静应力强度储备系数计算中，通常选取材料 的屈服极限作为极限应力 ${ }^{[19]}$ 。根据第四强度理论, 塑性材料的许用应力如下

$$
[\sigma]=\frac{\sigma_{\mathrm{s}}}{K}
$$

式中, $\sigma_{\mathrm{s}}$ 为材料屈服极限; $K$ 为安全系数, 取值范 围是 $1.5 \sim 2.0$ 。

该风机选用铝合金为叶轮材料，屈服极限 $\sigma_{\mathrm{s}}=280 \mathrm{MPa}$, 为保证模型的安全性, 取 $K=2$, 计算 得叶片许用应力 $[\sigma]=140 \mathrm{MPa}$, 而静力学结构分析中 三种方案最大等效应力仅为 $20.443 \mathrm{MPa}$, 远小于许 用应力 $[\sigma]$, 因此前掠叶片满足材料强度要求, 不会 产生拉伸变形甚至断裂，保证工作安全可靠。

\section{5 噪声预估}

轴流风机的噪声主要来自气动噪声和机械噪 声, 其中包括旋转噪声和浴旋噪声在内的气动噪声 是主要部分, 且难以降低 ${ }^{[20]}$ 。图 10 为设计工况下 动叶区中间截面处的声功率级分布, 定义声源功率 级 $L_{W}=10 \lg \left(P / P_{0}\right)$, 式中 $P$ 为声功率, $P_{0}$ 为基准声 功率, 其值为 $10^{-12} \mathrm{~W}$ 。图 10 表明, 叶顶间隙及其 


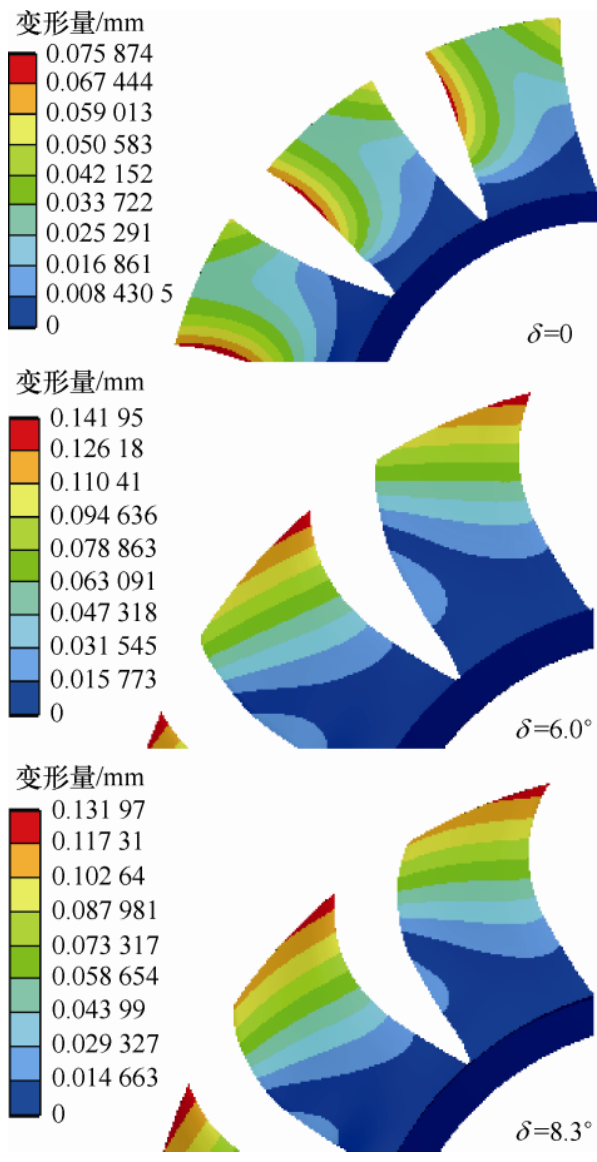

图 9 原叶片与前掠叶片的总变形分布

后端流道声功率级明显高于其他区域, 这主要是因 叶顶与机壳间存在微小间隙, 叶轮高速转动时, 叶 片吸/压力面侧形成压强差, 在此压强差和叶轮带动 的共同作用下, 叶顶间隙处产生泄漏流, 在间隙区 域内形成逆压梯度, 并干扰主流进一步形成二次流, 使得叶顶间隙及后端流场噪声显著 ${ }^{[21-22]}$; 流道靠近 压力面中下部声功率级最小, 这归因于叶轮对气流 的带动及流道内的回流现象共同作用, 并在叶轮旋 转作用下, 低噪声域沿逆旋转方向移动 ${ }^{[17]}$ 。图 10 显示, 原叶片与前掠叶片噪声分布差别不大, 但原 叶片最大声功率级为 $90 \mathrm{~dB}, \delta=6.0^{\circ}$ 和 $8.3^{\circ}$ 叶片最大 声功率级均为 $95 \mathrm{~dB}$, 较原风机提高 $5.56 \%$; 三种叶 片的最小声功率级并未发生变化, 为 $5 \mathrm{~dB}$, 且 $\delta=6.0^{\circ}$ 叶片最小声功率级分布区域面积较原风机 有所增加, $\delta=8.3^{\circ}$ 叶片最小声功率级区域面积较原 风机减小。综上所述, $\delta=6.0^{\circ}$ 和 $8.3^{\circ}$ 前掠叶片风机 较原风机最大声功率级增加, 最小声功率级不变, $\delta=8.3^{\circ}$ 时低噪声域面积较 $\delta=6.0^{\circ}$ 时有所减小, 整机 噪声相对较小, 但两种前掠叶片风机噪声较原风机 均有所提高, 因此在风机的实际运行中需采用积极 的降噪措施。

\section{6 改变动叶安装角}

综上所述, $\delta=8.3^{\circ}$ 叶片风机在全流量范围内性
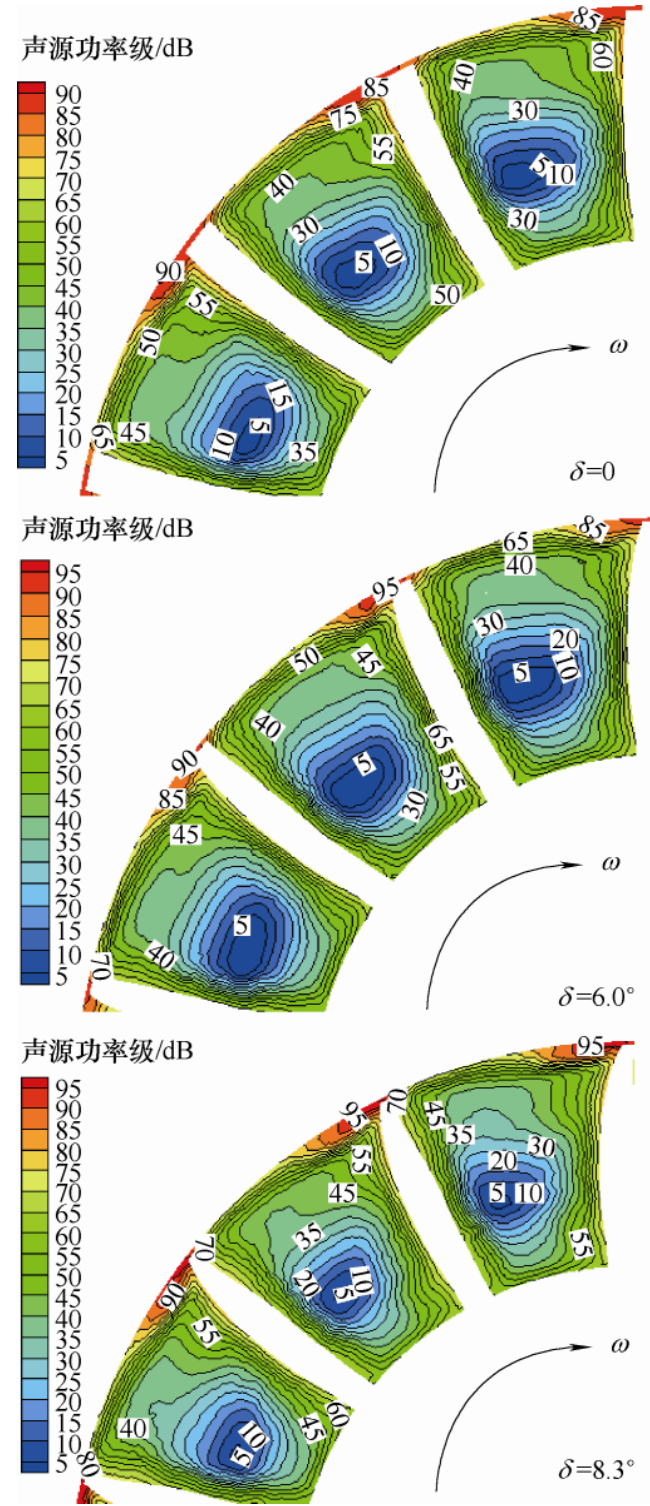

图 10 原叶片与前掠叶片流道内的噪声分布

能及噪声均优于 $\delta=6.0^{\circ}$ 情形, 因此下文探究在不同 动叶安装角下 $\delta=8.3^{\circ}$ 对风机性能的影响。

图 11 为三种安装角下原叶片与 $\delta=8.3^{\circ}$ 叶片风机 性能曲线。其中, 图 $11 \mathrm{~b}$ 为设计安装角情形, 具体 结果在本文 2.1 部分有详细说明, 此处不再赘述。 由图 $11 \mathrm{a}$ 和 $\mathrm{c}$ 可知, $\beta=29$ 和 $35^{\circ}$ 时, $\delta=8.3^{\circ}$ 叶片风机 全压在全流量范围较原风机均有所下降, 平均偏差 分别为 $-4.23 \%$ 和 $-2.7 \%$; 对于效率, $\beta=29^{\circ}$ 时, $\delta=8.3^{\circ}$

叶片风机效率在全流量范围较原风机平均降低 $1.02 \%, \beta=35^{\circ}$ 时, $Q=0.2 \sim 0.26$ 范围内, 前掠叶片 风机效率平均提升 $1.07 \%, Q=0.26 \sim 0.29$ 范围内平 均下降 $0.56 \%$ 。上述结果表明, $\beta=29^{\circ}$ 和 $35^{\circ}$ 情形下, $\delta=8.3^{\circ}$ 时风机性能有不同程度下降, 即设计安装角 下, 所选取前掠角度范围内的最佳前掠角度对其他 安装角情况并不一定适用。但对于长期处于额定负 荷运行的该类风机, 采用 $\delta=8.3^{\circ}$ 的弦向掠叶片可作 为一种提高风机性能的有效措施加以考虑。 


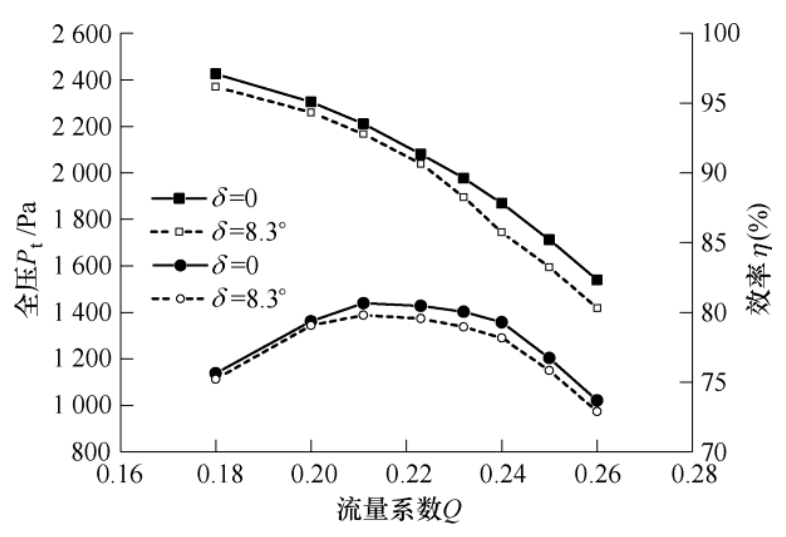

(a) $\beta=29^{\circ}$

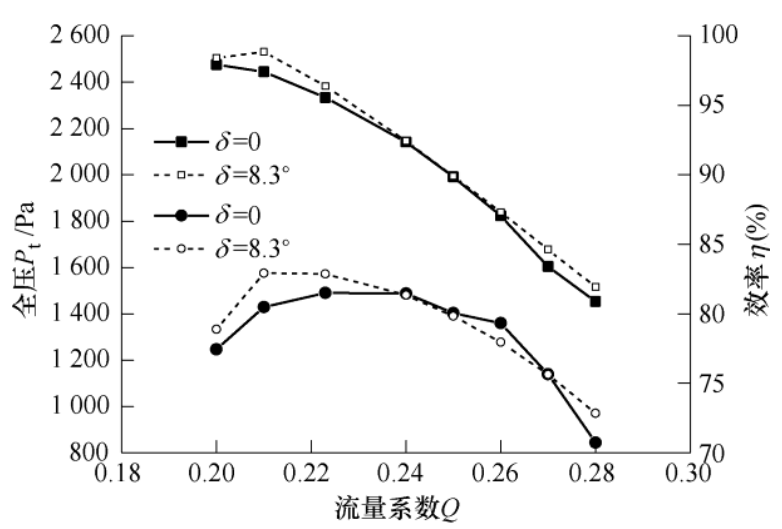

(b) $\beta=32^{\circ}$

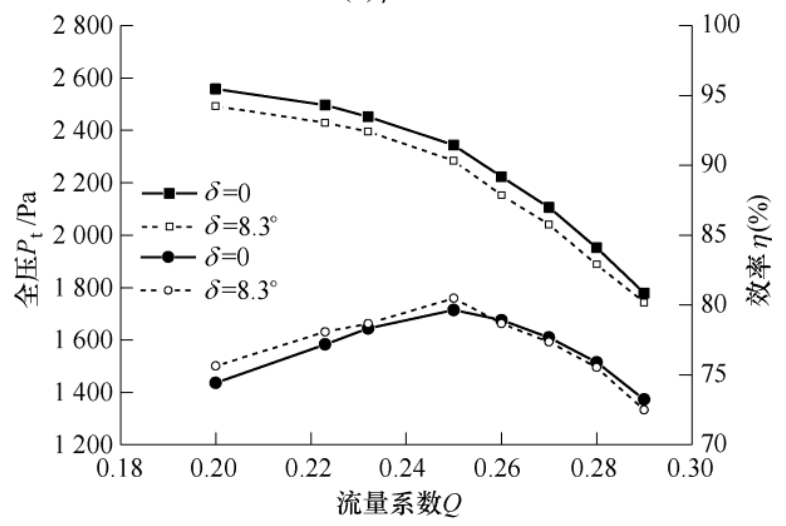

(c) $\beta=35^{\circ}$

图 $11 \beta=29^{\circ} 、 32^{\circ} 、 35^{\circ}$ 时直叶片与掠叶片风机性能曲线

\section{3 结论}

(1) 前掠叶片能有效提高动叶可调轴流风机的 气动性能, 在设计流量附近前掠 $8.3^{\circ}$ 为推荐角度, 计算的流量范围内的全压和效率平均提高 $1.85 \%$ 和 $0.64 \%$, 设计流量下全压和效率分别提高 $2.1 \%$ 和 $1.68 \%$ 。

(2) $\delta=8.3^{\circ}$ 叶片风机动叶区总压升系数和导叶 扩压系数较原风机均有所提高, 叶顶截面熵产率降 低, 可提升叶片做功能力, 降低叶顶泄漏损失, 提 高设计流量下的效率, 改善效果优于 $\delta=6.0^{\circ}$ 情形。 但两种方案最大声功率级较原风机提高 $5.56 \%$, 实
际运行中应采取积极的降噪措施。

(3) 静力结构分析结果表明: 前掠叶片较原叶 片最大等效应力和最大变形量较原叶片提高, 但仍 满足材料强度要求, 即前掠叶片不会产生拉伸变形 甚至断裂。

（4）最佳前掠角度 $\delta=8.3^{\circ}$ 在 $\beta=29^{\circ}$ 和 $35^{\circ}$ 情形下 的风机性能均有不同程度下降，因此对于长期带额 定负荷运行的该类风机，可采用 $\delta=8.3^{\circ}$ 的弦向掠叶 片作为一种提高风机性能的有效措施加以考虑。

\section{参 考 文 献}

[1] MOHAMMED K P, RAJ D P. Investigations on axial flow fan impellers with forward swept blades[J]. ASME Journal of Fluids Engineering, 1977, 99(3): 543-547.

[2] WADIA A R, SZUCS P N, CRALL D W. Inner workings of aerodynamic sweep[J]. ASME Journal of Turbomachinery, 1998, 120(4): 671-682.

[3] 金永平, 刘德顺, 文泽军. 矿用对旋式轴流通风机前后 两级叶片弯掠参数优化设计 $[\mathrm{J}]$. 煤炭学报, 2010 , 35(10): 1754-1759.

JIN Yongping, LIU Deshun, WEN Zejun. Optimization design for skew and sweep parameters of mine contra-rotating axial fan two-stage blades[J]. Journal of China Coal Society, 2010，35(10): 1754-1759.

[4] PATEL A B, VISWANATH K, NATH D D. Effect of axial sweep and tip extension on performance of an axial fan[C]//ASME 2016 International Mechanical Engineering Congress and Exposition. American Society of Mechanical Engineers , 2016 : V001T03A059V001T03A059.

[5] ILIKAN A N, AYDER E. Influence of the sweep stacking on the performance of an axial fan[J]. Journal of Turbomachinery, 2015, 137(6): 061004.

[6] MASI M, LAZZARETTO A. A simplified theory to justify forward sweep in low hub-to-tip ratio axial fan[C]//ASME Turbo Expo 2015: Turbine Technical Conference and Exposition. American Society of Mechanical Engineers, 2015: V001T09A011- V001T09A011.

[7] GIESECKE D, FRIEDRICHS J, STARK U, et al. Aerodynamic and acoustic performance of a single stage axial fan with extensive blade sweep designed to limit noise emissions[C]//ASME Turbo Expo 2016: Turbomachinery Technical Conference and Exposition. American Society of Mechanical Engineers, 2016: V001T09A004.

[8] CUI Weiwei, XIANG Xiaorong, ZHAO Qingjun, et al. The effect of sweep on flow fields of a highly loaded transonic rotor[J]. Aerospace Science and Technology, 2016, 58: 71-81. 
[9] BENINI E, BIOLLO R. Aerodynamics of swept and leaned transonic compressor-rotors[J]. Applied Energy, 2007, 84(10): 1012-1027.

[10] 昌皓, 朱芳, 金东海, 等. 叶片掠对跨声转子进口流动 的影响 [J]. 航空动力学报, 2016, 31(4): 857-866.

CHANG Hao, ZHU Fang, JIN Donghai, et al. Effect of blade sweep an inlet flow in transonic compressor rotors[J]. Journal of Aerospace Power, 2016, 31(4): 857-866.

[11] 宋彦萍, 刘振德, 赵桂杰, 等. 弯-掠叶片对压气机叶 栅端壁流动的控制作用 [J]. 推进技术，2004，25(4): 338-342.

SONG Yanping, LIU Zhende, ZHAO Guijie, et al. Effect of blade sweeping-curving on the endwall flow field of compressor cascade[J]. Journal of Propulsion Technology, 2004, 25(4): 338-342.

[12] 蔡娜, 李地. 弯掠动叶气动-声学优化设计及试验研究 [J]. 上海交通大学学报, 1997, 31(2): 81-85.

CAI Na, LI Di. Optimum design and experiment on skewed-swept rotating blades[J]. Journal of Shanghai Jiaotong University, 1997, 31(2): 81-85.

[13] 李春曦, 李新颖, 叶学民. 叶片切割对轴流风机性能影 响的数值研究 [J]. 机械工程学报, 2013, 50(10): 183-190.

LI Chunxi, LI Xinying, YE Xuemin. Numerical investigation of blade trimming effect on performance of an axial flow fan[J]. Journal of Mechanical Engineering, 2013, 50(10): 183-190.

[14] 崔建光. 叶顶间隙形态对轴流风机性能影响的数值研 究[D]. 北京: 华北电力大学, 2017.

CUI Jianguang. Numerical investigation of blade tip clearance patterns effect on performance of an axial flow fan[D]. Beijing: North China Electric Power University, 2017.

[15] 毛军, 杨立国, 郗艳红. 大型轴流风机叶片的气动弹性 数值分析研究 [J]. 机械工程学报, 2009, 45(11): 133-139.
MAO Jun, YANG Liguo, XI Yanhong. Numeric analysis of on the pneumatic elasticity of large axial flow fan blade[J]. Journal of Mechanical Engineering, 2009. 45(11): $133-139$.

[16] 张否, 郎进花, 王松岭. 电站轴流风机旋转失速工况下 的叶轮静力特性研究 $[\mathrm{J}]$. 动力工程学报, 2015, 35(5): 387-393.

ZHANG Lei, LANG Jinhua, WANG Songling. Static characteristics of the impeller of an axial flow fan under rotating stall conditions[J]. Journal of Power Engineering, 2015, 35(5): 387-393.

[17] YE Xuemin, LI Pengmin, LI Chunxi, et al. Numerical investigation of blade tip grooving effecton performance and dynamics of an axial flow fan[J]. Energy, 2015, 82: 556-569.

[18] LUAN H, WENG L, LUAN Y, et al. Numerical study on aerodynamic noise performances of axial spacing in a contra-rotating axial fan[J]. Journal of Vibroengineering, 2016, 18(8): 5605-5618.

[19] STARZMANN R, CAROLUS T. Effect of blade skew strategies on the operating range and aeroacoustic performance of the wells turbine[J]. Journal of Turbomachinery, 2014, 136(1): 011003.

[20] ZHANG L, WANG R, WANG S. Simulation of broadband noise sources of an axial fan under rotating stall conditions[J]. Advances in Mechanical Engineering, 2014, 6: 507079.

[21] LILLEY G M. The radiated noise from isotropic turbulence[J]. Theoretical and Computational Fluid Dynamics, 1994, 6(5-6): 281-301.

[22] FILIOS A E, TACHOS N S, FRAGIAS A P, et al. Broadband noise radiation analysis for an HAWT rotor[J]. Renewable Energy, 2007, 32(9): 1497-1510.

作者简介: 李春㬢(通信作者)女, 1973 出生, 博士, 教授。主要从事流 体机械、流体动力学理论及应用研究。

E-mail: leechunxi@163.com 